\title{
Projeto, fabricação e caracterização de uma bomba Tesla
}

\author{
Diego Hayashi Alonso, Orientador: Prof. Dr. Emílio Carlos Nelli Silva
}

\begin{abstract}
Resumo - Dispositivos Tesla são compostos de discos rotativos (sem palhetas) que funcionam por efeito da camada limite (i.e., por forças de atrito viscoso atuando no fluido e efeito Coandă). Segundo esse princípio de funcionamento, o fluxo resulta contínuo e não pulsátil. O objetivo deste projeto é projetar, fabricar e caracterizar uma bomba Tesla visando melhorar eficiência e potência. A bomba Tesla tem várias aplicações, porém a eficiência de seu funcionamento é consideravelmente baixa, o que abre espaço para otimização do seu projeto. Este projeto é realizado por meio de otimização do rotor e da voluta da bomba Tesla para operação com um fluido newtoniano em escoamento laminar. O funcionamento da bomba Tesla é simulado usando o Método de Elementos Finitos na plataforma FEniCS e validado com o software ANSYS ${ }^{\circledR}$ CFX. A otimização topológica é implementada na plataforma FEniCS utilizando-se a biblioteca dolfin-adjoint no cálculo de sensibilidades (derivadas) e o algoritmo IPOPT para otimização. São fabricados e caracterizados experimentalmente dois protótipos: um protótipo de bomba Tesla usando CDs (discos de policarbonato usados para armazenamento digital de dados) como os discos do rotor, com o espaçamento otimizado por análise paramétrica; e um protótipo projetado pelo método de otimização topológica (fabricado por meio de manufatura aditiva).
\end{abstract}

Palavras-chave - Bombas centrífugas; Dinâmica dos fluidos (Simulação); Método dos elementos finitos; Métodos topológicos (Otimização).

\section{Introdução}

Uma bomba Tesla é uma bomba centrífuga de múltiplos discos rotativos. Em uma bomba Tesla, o fluido entra sem rotação e é propulsionado pelo efeito da camada limite em discos paralelos espaçados girando ao redor de um eixo, sem a necessidade de palhetas. Na configuração convencional, o motor é acoplado ao eixo da bomba Tesla e o escoamento entra axialmente e sai radialmente (ver figura 1). Neste texto, por simplicidade, princípio da bomba Tesla é referido como "Princípio Tesla".

Versão inicial submetida em 02 de Fev. de 2018. Versão final aceita em 26 de Jun. de 2018. Publicado em 01 de Dez. de 2018. Digital Object Identifier 10.11606/issn.2526-8260.mecatrone.2018.143554 
Figura 1 - Bomba Tesla segundo a patente inicial de Nikola Tesla
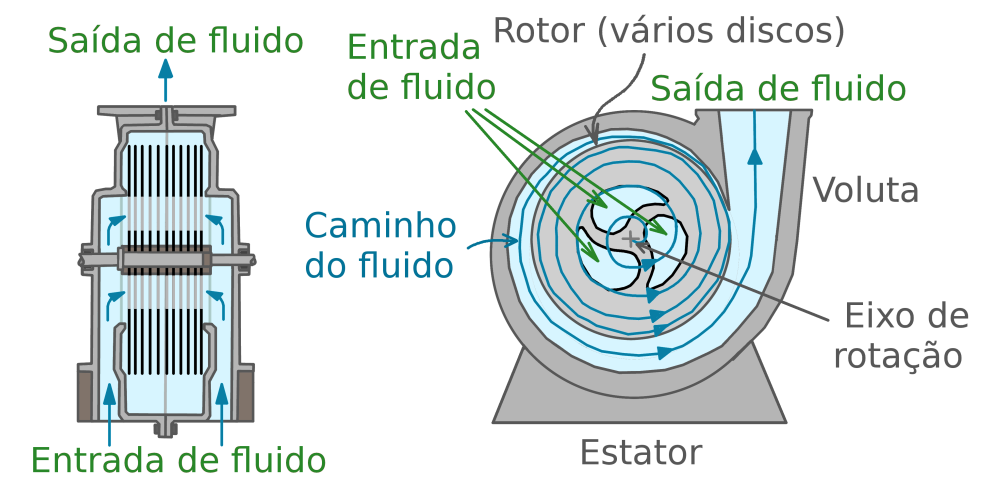

Fonte: Baseado em Podergajs (2011, p. 3) e Tesla (1913a, p. 1)

O escoamento em uma bomba Tesla se assemelha ao escoamento quando uma placa plana é movida tangencialmente em relação a outra, ocasionando a formação de uma camada limite e um escoamento característico (escoamento de Couette). A principal diferença é que, para uma bomba Tesla, as placas estão em rotação. A formação da camada limite está ilustrada na figura 2.

Figura 2 - Formação da camada limite em dois casos distintos: separadas e juntas (escoamento plenamente desenvolvido)

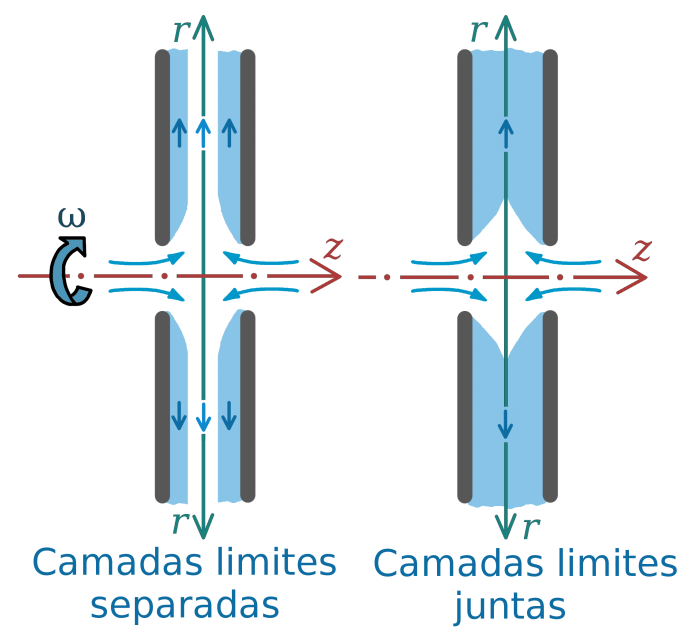

Fonte: Baseado em Dodsworth (2016, p. 31)

Como resultado da rotação dos discos, o escoamento é essencialmente espiral. Dependendo da relação entre as velocidades radial e tangencial, as voltas da espiral podem resultar mais próximas ou mais espaçadas.

O princípio Tesla pode ser aplicado para bomba ou turbina (TESLA, 1913b, p. 1). A operação como turbina é caracterizada por baixa eficiência em aplicações de alta potência (RAJE et al., 2015, p. 29), o que limita o seu uso comercial. Porém, a sua utilização em baixas potências resulta em mais eficiência do que as turbinas convencionais (RAJE et al., 2015, p. 29).

O princípio Tesla possui diversas aplicações e em vários casos: turbinas a vapor (RAJE et al., 2015, p. 30 e 31), casos em que o fluido de trabalho contém particulados (como sal e água contaminada) (RAJE et al., 2015, p. 30 e 31), casos com fluidos de baixa e alta viscosidades (RAJE et al., 2015, p. 30 e 31), DAVs (Dispositivos de Assistência Ventricular) (YU, 2015, p. 4), cogeração em micro-plantas de geração de energia (LAMPART et al., 2009), sistemas pico-hidráulicos para eletrificação rural (HO-YAN, 2011) etc. 
O princípio Tesla também pode ser estendido para aplicações com gases, como turbinas a gás e vapor (TESLA, 1921b), ventiladores (ENGIN et al., 2009), turbinas eólicas (FULLER, 1913), compressores (RICE, 1991) e até mesmo na geração de vácuo (TESLA, 1921a).

Apesar de bombas operando sob o princípio Tesla ainda não possuírem amplo uso comercial (MEDVITZ et al., 2011, p. 2), o princípio Tesla possui diversas características atrativas com relação às bombas de palhetas (IZRAELEV et al., 2009, p. 556) (MEDVITZ et al., 2011, p. 2): manufatura e montagem mais simples (custo menor), tensões de escoamento turbulento menores (menor turbulência), ausência de condições de cavitação, escoamento mais suave de fluido (quantidade de movimento linear do fluido varia lentamente) e força mais uniforme sobre o fluido (não há força gerada pela palheta ao passar pela saída de uma bomba Tesla). Além disso, o efeito de vibração na eficiência é muito pequeno (DODSWORTH; GROULX, 2015) .

Segundo Dodsworth (2016, p. 1), o princípio Tesla resulta em menor sensibilidade à cavitação induzida por vibração do que bombas centrifugas convencionais (RODDY et al., 1987), e funciona bem com fluidos viscosos, o que se mostra vantajoso em aplicações de resfriamento (DODSWORTH, 2016, p. 1) e para fluidos de trabalho voláteis, como combustíveis líquidos (DODSWORTH, 2016, p. 5). Existe também a possibilidade de utilização da bomba Tesla em DAVs (Dispositivos de Assistência Ventricular, VADs, Ventricular Assist Devices) (i.e., bombas de sangue), porque o escoamento resulta contínuo e não pulsátil, o que pode reduzir danos ao sangue: hemólise e trombose (BEHBAHANI et al., 2009, p. 5). Porém, a eficiência da bomba Tesla é relativamente baixa (normalmente menor do que $20 \%$ (YU, 2015, p. 4) (FOSTER, 2006, p. 280)), o que gera grande transferência de calor de calor para o sangue, havendo a possibilidade de se tornar um problema para o sangue e a saúde do paciente (YU, 2015, p. 4). Portanto, há necessidade de aumentar essa eficiência.

Existem basicamente três tipos de métodos de otimização: paramétrica, de forma e topológica, sendo que este último é o mais genérico. O método de otimização topológica consiste em distribuir fluido / sólido em um dado domínio de projeto. Existem basicamente duas formas de implementálo: "método de densidade" (BENDSØE; KIKUCHI, 1988) (BORRVALL; PETERSSON, 2003) e "level-set" (OSHER; SETHIAN, 1988) (DUAN et al., 2016).

O objetivo deste projeto é modelar, simular, projetar e otimizar o rotor e a voluta de uma bomba Tesla com um software de elementos finitos, de modo a aumentar a sua eficiência, e fabricar e caracterizar dois protótipos: um protótipo de bomba Tesla de CDs (discos de policarbonato usados para armazenamento digital de dados) projetado por análise paramétrica, e um protótipo projetado pelo método de otimização topológica (fabricado por meio de manufatura aditiva).

Como a aplicação de otimização topológica em domínio 3D resulta em custo computacional relativamente alto se comparado a otimização topológica em domínios $2 \mathrm{D}$, utilizaram-se: modelo $2 \mathrm{D}$ de escoamento girante para projeto do rotor, e modelo $2 \mathrm{D}$ comum para projeto da voluta.

Este trabalho está organizado da seguinte forma: na seção 2, é descrita a fundamentação teórica; na seção 3, é descrita a implementação numérica de simulação e otimização topológica na plataforma FEniCS; na seção 4, é apresentada a metodologia experimental; na seção 5, são apresentados os resultados numéricos e experimentais; na seção 6, são inferidas algumas conclusões.

\section{Fundamentação teórica}

Para modelagem do escoamento de fluido, foram consideradas as equações da continuidade e de quantidade de movimento linear (Navier-Stokes). As principais hipóteses são: escoamento laminar, fluido incompressível e variações desprezíveis de viscosidade. 


\subsection{Equações de equilíbrio}

As equações de equilíbrio são consideradas em referencial rotativo, em que aparecem forças de Coriolis e centrípeta. Também considera-se o modelo de Brinkman para meio poroso (VAFAI, 2005, p. 110) (para otimização topológica), que implica que, dentro do meio poroso, surge uma força de resistência diretamente proporcional à velocidade do fluido em relação ao material ( $\left.\boldsymbol{f}_{r}=-\kappa(\alpha) \boldsymbol{v}_{\text {mat }}\right)$. Assim, as equações do problema são:

$\nabla \cdot \boldsymbol{v}_{\mathrm{abs}}=0$

$\rho \nabla \boldsymbol{v} \bullet \boldsymbol{v}=\nabla \cdot \boldsymbol{T}+\rho \boldsymbol{f}-2 \rho(\boldsymbol{\omega} \wedge \boldsymbol{v})-\rho \boldsymbol{\omega} \wedge(\boldsymbol{\omega} \wedge \boldsymbol{s})-\kappa(\alpha) \boldsymbol{v}_{m a t}$

em que $\boldsymbol{v}$ é a velocidade relativa, $\boldsymbol{s}$ é a posição, $\rho$ é a densidade do fluido, $p$ é a pressão, $\mu$ é a viscosidade dinâmica, $\rho \boldsymbol{f}$ é a força por unidade de volume atuando no fluido, $\kappa(\alpha)$ é o coeficiente de absorção ("permeabilidade inversa"), $\alpha$ é a pseudo-densidade (variável de projeto, em que possui valores entre 0 (sólido) e 1 (fluido)), $\boldsymbol{v}_{\text {mat }}$ é a velocidade em relação ao meio poroso, e $\boldsymbol{T}$ é o tensor de tensões dado por

$$
\boldsymbol{T}=2 \mu \boldsymbol{\epsilon}-p \boldsymbol{I}, \boldsymbol{\epsilon}=\frac{1}{2}\left(\nabla \boldsymbol{v}+\nabla \boldsymbol{v}^{T}\right)
$$

O rotor pode ser simplificado para modelo 2D de escoamento girante ("modelo 2D axissimétrico com giro"), e a voluta pode ser simplificada para o modelo 2D comum (figura 3).

Figura 3 - Modelos utilizados nos projetos do rotor e da voluta da bomba Tesla
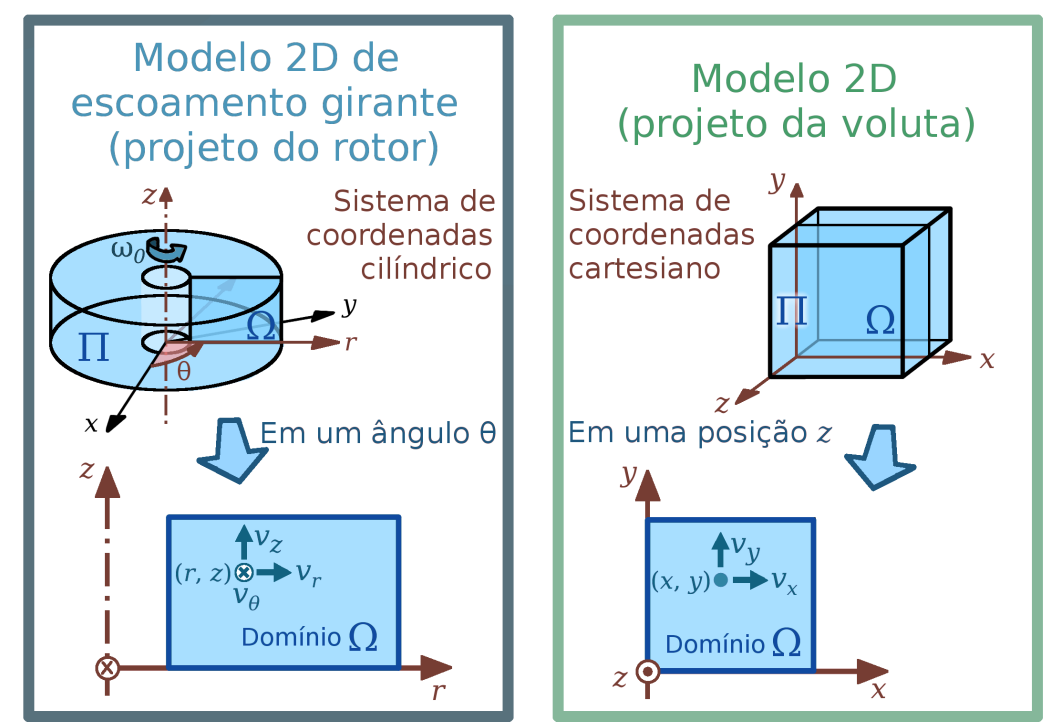

Fonte: Própria

No modelo 2D de escoamento com giro, considerando-se um sistema de coordenadas cilíndrico,

$\boldsymbol{s}=(r, 0, z)=r \boldsymbol{e}_{r}+z \boldsymbol{e}_{z}$

$\boldsymbol{v}=\left(v_{r}, v_{\theta}, v_{z}\right)=v_{r} \boldsymbol{e}_{r}+v_{\theta} \boldsymbol{e}_{\theta}+v_{z} \boldsymbol{e}_{z}$

Assumindo-se axissimetria, as derivadas com relação a $\theta$ se tornam zero (i.e., $\frac{\partial()}{\partial \theta}=0$ ). 


\subsection{Modelo de material}

Borrvall e Petersson (2003, p. 96), sugere a seguinte função de interpolação convexa como modelo de material, para "relaxar" o problema de otimização topológica

$\kappa(\alpha)=\kappa_{m a ́ x}+\left(\kappa_{m i ́ n}-\kappa_{m a ́ x}\right) \alpha \frac{1+q}{\alpha+q}$

em que $\kappa_{m i ́ n}$ e $\kappa_{m a ́ x}$ são o mínimo e máximo valores de $\kappa(\alpha)$. O parâmetro $q$ é o parâmetro de penalização, que controla a convexidade de $\kappa(\alpha)(q>0)$.

\subsection{Método de elementos finitos}

A forma fraca das equações de equilíbrio são dadas pelo Método dos Resíduos Ponderados (MRP) e pelo método de Galerkin na forma de uma formulação mista de velocidade-pressão. Assim, para cada nó $j$ de interpolação de cada elemento, no caso do rotor,

$$
\begin{aligned}
R_{c, j} & =\int_{\Omega}[\nabla \cdot \boldsymbol{v}] \boldsymbol{w}_{p, j} r d \Omega \\
R_{m, j} & =\int_{\Omega}[\rho \nabla \boldsymbol{v} \bullet \boldsymbol{v}-\rho \boldsymbol{f}+2 \rho(\boldsymbol{\omega} \wedge \boldsymbol{v}) \rho \boldsymbol{\omega} \wedge(\boldsymbol{\omega} \wedge \boldsymbol{s})] \bullet \boldsymbol{w}_{v, j} r d \Omega+\int_{\Omega} \boldsymbol{T} \bullet\left(\nabla \boldsymbol{w}_{v, j}\right) r d \Omega \\
& -\oint_{\Gamma}\left(\boldsymbol{T} \bullet \boldsymbol{w}_{v, j}\right) \bullet \boldsymbol{n} r d \Gamma+\int_{\Omega} \boldsymbol{\kappa}(\alpha) \boldsymbol{v}_{m a t} \bullet \boldsymbol{w}_{v, j} r d \Omega
\end{aligned}
$$

em que o índice subscrito $c$ significa "equação da continuidade" e o índice subscrito $m$ significa "equação da quantidade de movimento linear" (equações de Navier-Stokes), as funções peso são $w_{p, j}$ (para pressão no rotor/voluta) e $\boldsymbol{w}_{v, j}=\left[\begin{array}{l}w_{v, j, r} \\ w_{v, j, \theta} \\ w_{v, j, z}\end{array}\right]$ (para velocidade).

Para a voluta, as equações são as mesmas, mas a velocidade, posição e função peso de velocidade têm somente duas componentes $(\mathrm{x}, \mathrm{y})$ e não há o fator multiplicativo $r$ das equações (6) e (7).

\subsection{Escolha do elemento finito}

Para estabilidade da formulação velocidade-pressão, a escolha comum é usar elementos de Taylor-Hood (figura 4): interpolação de grau 1 para pressão (elemento $P_{1}$ ) e de grau 2 para velocidade (elemento $P_{2}$ ). Para a variável de projeto, foi escolhida interpolação de grau 1 (elemento $\left.P_{1}\right)$.

Figura 4 - Escolha de elementos fnitos para pressão, velocidade e variável de projeto
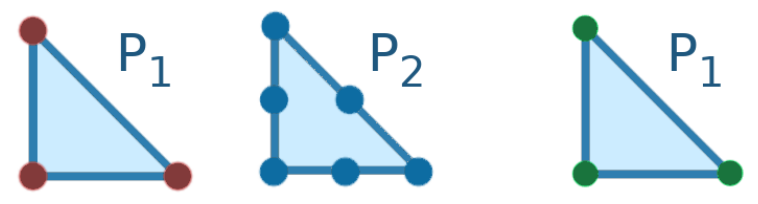

Pressão $p$ Velocidade $\boldsymbol{v}$ Variável de projeto $\alpha$

Fonte: Própria 


\subsection{Otimização topológica} volume.

O problema de otimização topológica está definido abaixo, sujeito a uma restrição de

$\min _{\alpha} J(p(\alpha), \boldsymbol{v}(\alpha), \alpha)$

tal que

Equações de equilíbrio + Condições de contorno

Restrição de volume de fluido: $\int_{\Omega_{\alpha}} \alpha\left(2 \pi r d \Omega_{\alpha}\right) \leqslant f V_{0}$ (rotor)

$$
\int_{\Omega_{\alpha}} \alpha d \Omega_{\alpha} \leqslant f V_{0} \text { (voluta) }
$$

Restrição de caixa, de $\alpha: 0 \leqslant \alpha \leqslant 1$

Seguindo-se a abordagem de otimização topológica de (BORRVALL; PETERSSON, 2003) para minimização de perda de carga em canais, pode-se utilizar a dissipação de energia relativa como função objetivo:

$J(p(\alpha), \boldsymbol{v}(\alpha), \alpha)=\Phi_{r e l}=\int_{\Omega}\left[\frac{1}{2} \mu\left(\nabla \boldsymbol{v}+\nabla \boldsymbol{v}^{T}\right) \bullet\left(\nabla \boldsymbol{v}+\nabla \boldsymbol{v}^{T}\right)-\rho \boldsymbol{f} \bullet \boldsymbol{v}\right] 2 \pi r d \Omega+\int_{\Omega} \kappa(\alpha) \boldsymbol{v}_{m a t} \bullet \boldsymbol{v} 2 \pi r d \Omega$

Para a voluta, considera-se a dissipação de energia absoluta, que utiliza velocidade absoluta $\left(\boldsymbol{v}_{\text {abs }}\right)$, em coordenadas cartesianas 2D.

\subsection{Análise de semelhança}

Para a voluta, definiu-se um número de Reynolds local com base no comprimento aproximado do canal ao redor do rotor, e na velocidade máxima de escoamento,

$\operatorname{Re}_{\ell}=\frac{\left|\boldsymbol{v}_{\mathrm{abs}}\right| \ell}{\nu}, \ell=2 \pi\left(r_{\text {ext }}+\frac{b_{\text {saída }}}{2}\right)+\left(x_{\text {rotor }}-r_{\text {ext }}\right)$

em que $\left|\boldsymbol{v}_{\text {abs }}\right|$ é o módulo da velocidade absoluta do fluido, $\nu$ é a viscosidade cinemática, $r_{\text {ext }}$ é o raio externo (saída) da do rotor da bomba, $x_{\text {rotor }}$ é a distância horizontal do centro da bomba com relação à saída do canal, e $b_{\text {saída }}$ é a largura do canal na saída.

Os parâmetros adimensionais selecionados para análise do rotor da bomba Tesla estão na tabela 1, em que as suas definições originais foram adaptadas para possibilitar a análise de resultados de otimização topológica. 
Tabela 1 - Parâmetros adimensionais para análise do rotor

\begin{tabular}{cc}
\hline Parâmetro adimensional & Equação * \\
\hline Caracterização da camada limite & \\
\hline
\end{tabular}

Parâmetro de Pohlhausen

(REY LADINO, 2004, p. 28) (BREITER; POHLHAUSEN, 1962, p. 11)

$\mathrm{Ph}=e_{\min } \sqrt{\frac{\omega}{\nu}}$

Caracterização do regime de escoamento

Número de Reynolds local com relação ao raio externo

$$
\operatorname{Re}_{\mathrm{ext}, \ell}=\frac{\left|\boldsymbol{v}_{\mathrm{abs}}\right| r_{\mathrm{ext}}}{\nu}
$$

Parâmetro viscogeométrico (de Nendl) local

(com base em Rey Ladino (2004, p. 27))

$$
\mathrm{Nd}_{\mathrm{ext}, \ell}=\frac{\left|\boldsymbol{v}_{\mathrm{abs}}\right| e_{\mathrm{min}}^{2}}{\nu r_{\mathrm{ext}}}
$$

Caracterização da vazão

\section{Essential machine data parameter}

(com base em Foo et al. (2010, p. 674)

$$
\mathrm{A}=\frac{Q e_{\mathrm{mín}}}{n_{\text {saídas }} \nu r_{\mathrm{int}}^{2}}
$$

Caracterização de eficiência

Eficiência isentrópica (rendimento isentrópico)

(baseado em (REY LADINO, 2004, p. 29))

$$
\eta_{s}=\frac{P_{\text {ideal }}}{P_{\text {real }}}=\frac{\Delta h_{s}}{P_{f} / \dot{m}}
$$

* Legenda:

$\omega$ : Velocidade angular $(\mathrm{rad} / \mathrm{s})$

$\nu$ : Viscosidade cinemática $\left(\mathrm{m}^{2} / \mathrm{s}\right)$

$\rho:$ Densidade $\left(\mathrm{kg} / \mathrm{m}^{3}\right)$

$e_{\text {mín }}:$ Espaçamento mínimo entre discos $(\mathrm{m})$

$r_{\text {int }}$ : Raio interno (entrada) do rotor da bomba $(\mathrm{m})$

$r_{\text {ext }}$ : Raio externo (saída) do rotor da bomba (m)

$\left|\boldsymbol{v}_{\text {abs }}\right|$ : Módulo da velocidade absoluta do fuido $(\mathrm{m} / \mathrm{s})$

$Q$ : Vazão volumétrica $\left(\mathrm{m}^{3} / \mathrm{s}\right)$

$\dot{m}$ : Vazão mássica $(\mathrm{kg} / \mathrm{s})$

$\Delta h_{s}=g H$ : Variação de entalpia específca (trabalho específco) no processo ideal $(\mathrm{J} / \mathrm{kg})$, em que $g$ é a aceleração da gravidade e $H$ é a carga manométrica.

$n_{\text {saídas }}$ : Número de saídas de fuido da bomba Tesla

\section{Implementação numérica}

A plataforma FEniCS (LOGG et al., 2012) é usada para implementar o Métodos de Elementos Finitos e Otimização Topológica, usando o método adjunto para o cálculo de sensibilidades (derivadas) (biblioteca dolfin-adjoint (FARRELL et al., 2013)) e algoritmo 
de otimização de ponto interior (IPOPT (WÄCHTER; BIEGLER, 2006)). A solução para o Método de Elementos Finitos usa o método de Newton-Raphson e o resolvedor ("solver") utilizado é o MUMPS (Multifrontal Massively Parallel sparse direct Solver) (AMESTOY et al., 2001).

O fluxograma da figura 5 mostra a interconexão entre os pacotes de software.

Figura 5 - Fluxograma do problema de otimização topológica

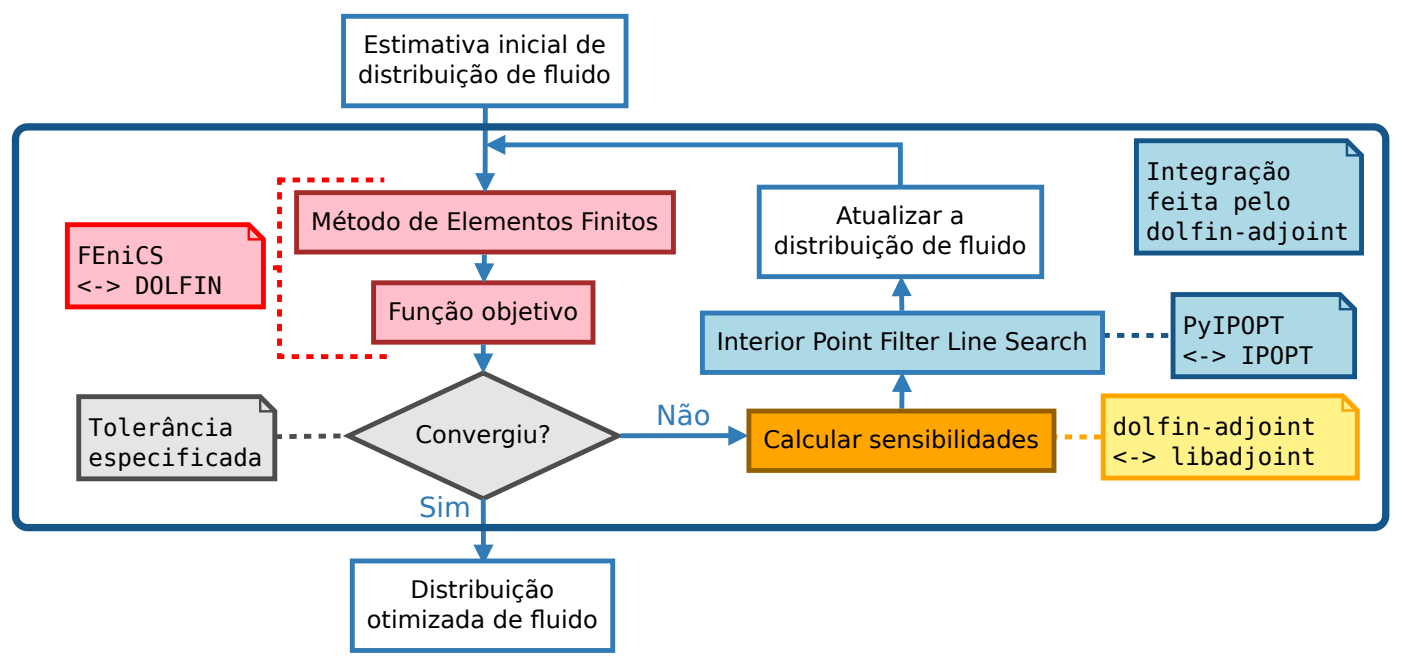

Fonte: Própria

\section{Metodologia experimental}

O projeto do protótipo da bomba Tesla de CDs está ilustrado na figura 6 e a bancada experimental está ilustrada na figura 7.

Figura 6 - Projeto da bomba Tesla de CDs

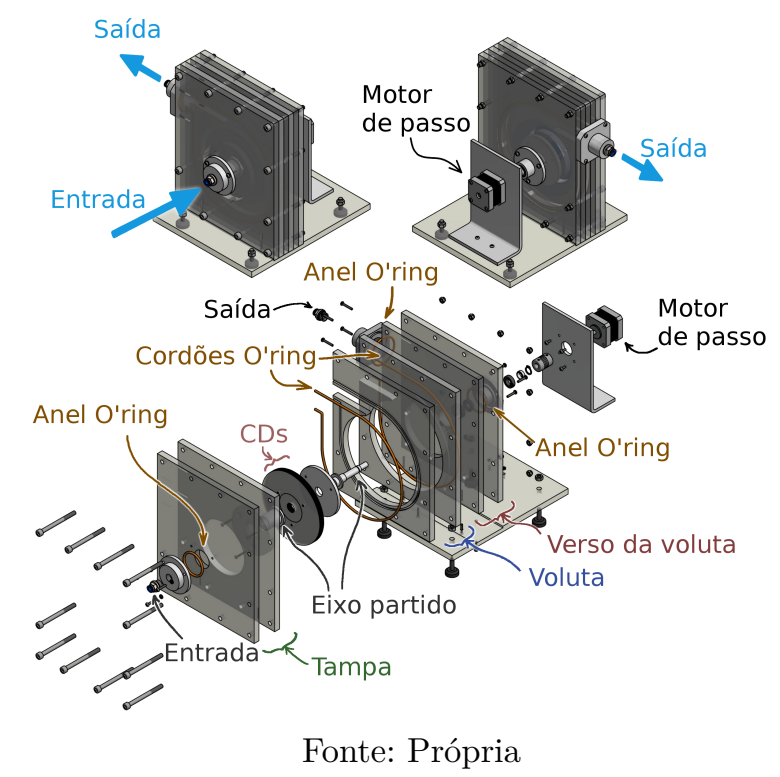


Figura 7 - Bancada experimental para a bomba Tesla de CDs

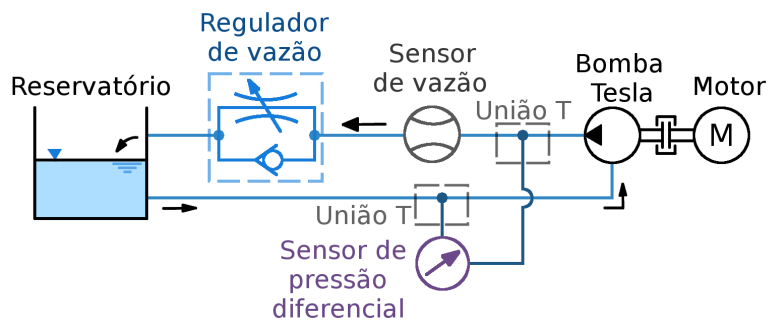

Fonte: Própria

O projeto do protótipo da bomba Tesla otimizada ("mini bomba Tesla") está ilustrado na figura 8 e a bancada experimental está ilustrada na figura 9.

Figura 8 - Projeto da mini bomba Tesla

(a) Rotor de discos retos

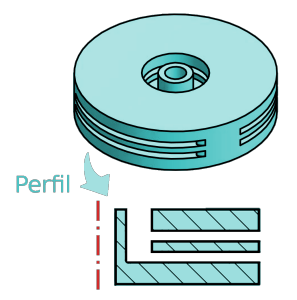

(b) Rotor otimizado para dissipação de energia

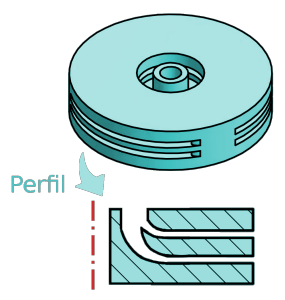

(c) Voluta comum

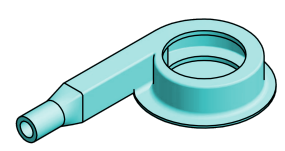

(d) Voluta otimizada para dissipação de energia

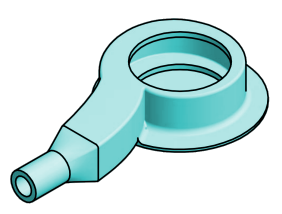

Fonte: Própria

Figura 9 - Bancada experimental para a mini bomba Tesla

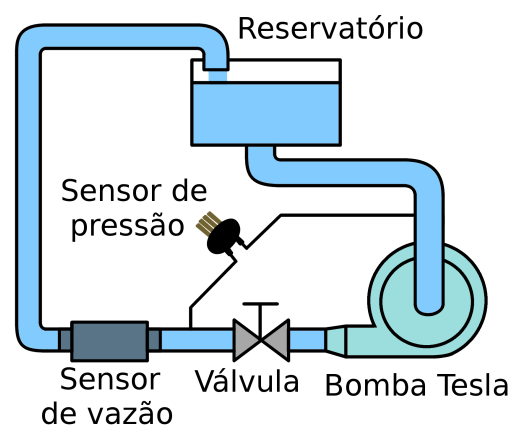

Fonte: Própria

\section{Resultados}

Os resultados numéricos consideram o fluido como sendo água ( $\mu=0,001 \mathrm{~Pa} \mathrm{~s}$ e $\left.\rho=1000,0 \mathrm{~kg} / \mathrm{m}^{3}\right)$. Para escalar as equações e aumentar a precisão de cálculo da forma fraca, funcionais e sensibilidades, utilizou-se o sistema MMGS (Milímetros-Gramas-Segundos) (i.e., unidades de comprimento e massa sendo multiplicadas por um fator $10^{3}$ ). Como utilizar propriedades da água piora muito a convergência do método de Newton-Raphson, utilizou-se um método de continuação, em que, enquanto a densidade se mantém em $1000,0 \mathrm{~kg} / \mathrm{m}^{3}$ desde o início, a viscosidade dinâmica começa como $100 \mathrm{~Pa}$ s, gradualmente reduzindo até 0,001 Pa s. O 
resultado de simulação com cada viscosidade dinâmica é usado como estimativa inicial para a simulação com a próxima viscosidade dinâmica menor.

Como forças de corpo como a gravidade são normalmente menores que outras forças envolvidas em uma máquina de fluxo, elas foram desprezadas $(\rho \boldsymbol{f}=(0,0,0))$.

O pós-processamento das topologias otimizadas foi feito com uma função degrau simples

$\alpha_{\text {pós-processado }}=\left\{\begin{array}{l}1 \text { (fluido) }, \text { se } \alpha \geqslant 0,5 \\ 0 \text { (sólido) }, \text { se } \alpha<0,5\end{array}\right.$

As malhas de elementos finitos para o rotor da bomba Tesla são estruturadas, com partições retangulares de 4 triângulos a partir das diagonais de cada retângulo.

\subsection{Análise paramétrica do rotor da bomba Tesla de CDs}

Foram utilizadas as dimensões de CDs $\left(r_{\text {int }}=7,5 \mathrm{~mm}\right.$ e $\left.r_{\text {ext }}=60 \mathrm{~mm}\right)$ para as simulações de escoamento entre dois discos rotativos a $500 \mathrm{rpm}$ e vazão de entrada de $0,5 \mathrm{~L} / \mathrm{min}$ (figura 10). A malha de elementos finitos é de $320 \times 20$ partições retangulares.

Figura 10 - Modelo para análise paramétrica do rotor da bomba Tesla de CDs

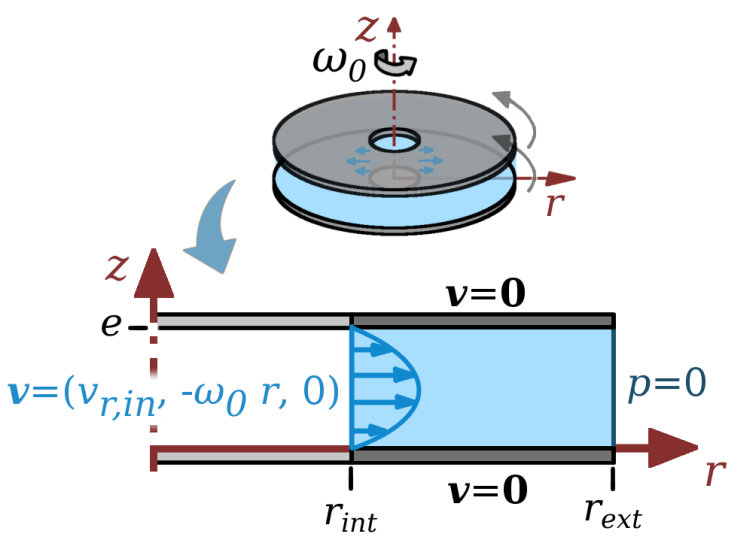

Fonte: Própria

Analisando-se a dissipação de energia em função do espaçamento entre discos (figura 11), percebe-se que o ponto de mínima dissipação de energia está em 0,55 mm.

Figura 11 - Dissipação de energia para cada espaçamento entre CDs

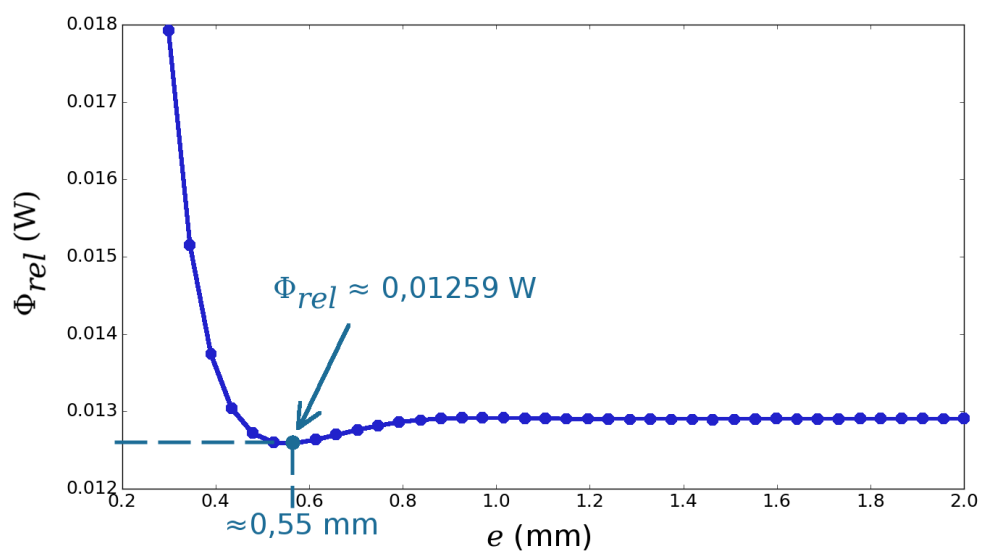

Fonte: Própria 
Para projeto da voluta da bomba Tesla de CDs, foram seguidas algumas diretrizes de Lobanoff e Ross (2013, p. 50 e 59) e Tuzson (2000, p. 145).

\subsection{Otimização topológica do rotor da bomba Tesla}

Para otimização do rotor da bomba Tesla, utilizaram-se três modelos diferentes: de entrada horizontal (igual à figura 10), de entrada vertical com um canal (figura 13), e de entrada vertical com dois canais (figura 13). O modelo de entrada vertical com dois canais considera repetição de padrão (ALMEIDA et al., 2010).

Figura 12 - Modelo para otimização de uma bomba Tesla de entrada vertical com um canal

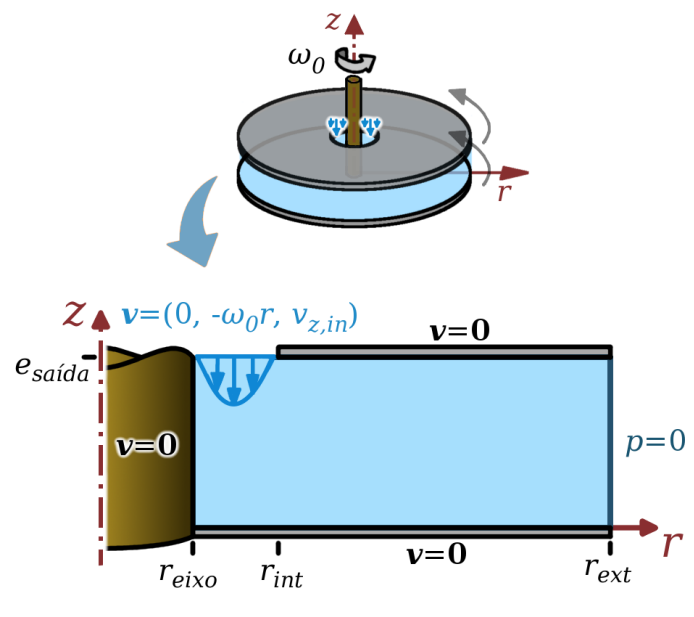

Fonte: Própria

Figura 13 - Modelo para otimização de uma bomba Tesla de entrada vertical com dois canais

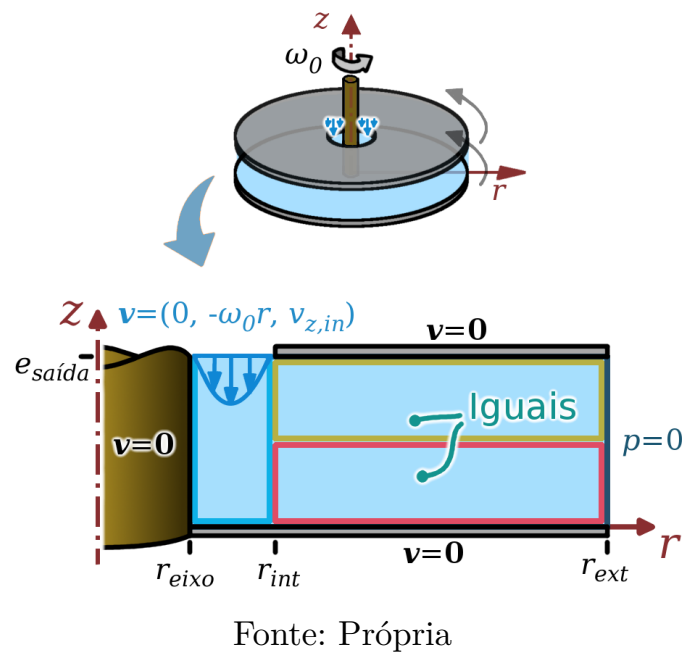

O efeito da rotação nas topologias otimizadas para os três modelos considerados está nas figuras 14, 15 e 16, considerando somente dissipação de energia e comparando com bombas Tesla de discos retos. A vazão considerada é de $0,5 \mathrm{~L} / \mathrm{min}$ e as dimensões são $r_{\text {int }}=5 \mathrm{~mm}$, $r_{\text {ext }}=15 \mathrm{~mm}, e=2 \mathrm{~mm}, r_{\text {eixo }}=2,6 \mathrm{~mm}$ e $e_{\text {saída }}=5 \mathrm{~mm}$. 
Figura 14 - Efeito da rotação para bomba Tesla de entrada horizontal

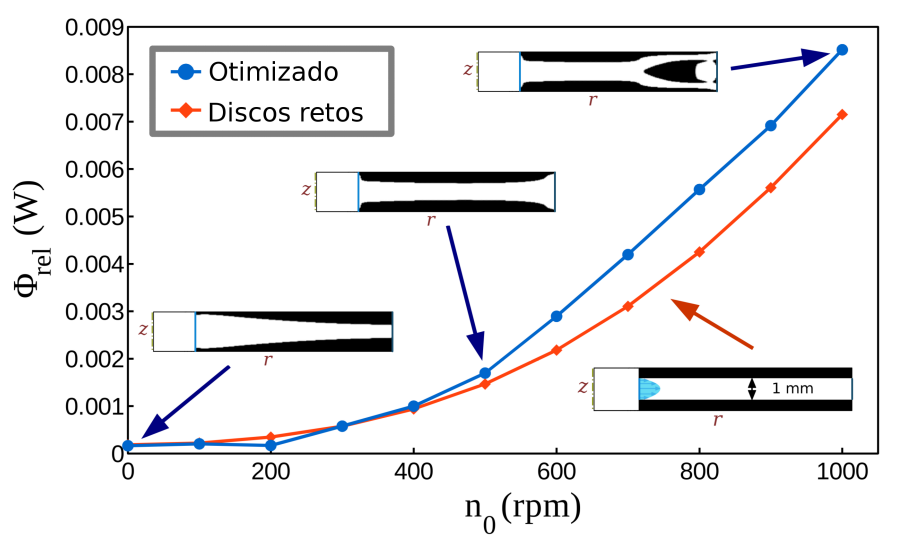

Fonte: Própria

Figura 15 - Efeito da rotação para bomba Tesla de entrada vertical com um canal

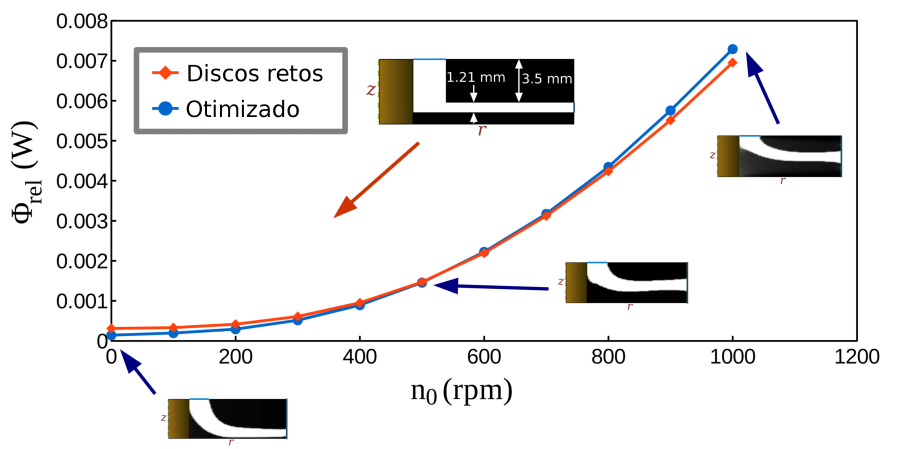

Fonte: Própria

Figura 16 - Efeito da rotação para bomba Tesla de entrada vertical com dois canais

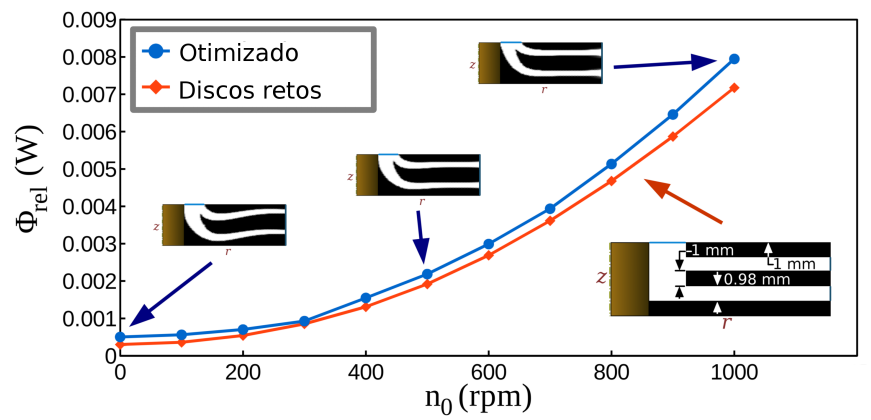

Fonte: Própria

Nas figuras 14, 15 e 16, cabe notar que os valores das topologias otimizadas são obtidos por meio de pós-processamento com um filtro simples, portanto as fronteiras ainda estão muito "rugosas" (devido à malha discreta), e a dissipação de energia deve ser maior do que seria se as fronteiras fossem "suaves" (como quando interpoladas por splines).

\subsection{Otimização topológica da voluta da bomba Tesla}

A voluta da bomba Tesla foi otimizada conforme o modelo da figura 17 ( $d_{\text {rotor }}=30 \mathrm{~mm}, \quad h=60 \mathrm{~mm}, \quad x_{\text {rotor }}=50 \mathrm{~mm}$ e $\left.y_{\text {rotor }}=30 \mathrm{~mm}\right)$, considerando-se veloci- 
dade radial $v_{r}=0,001 \mathrm{~m} / \mathrm{s}$ e $v_{\theta}=0,01 \mathrm{~m} / \mathrm{s}$. A topologia otimizada está indicada na figura 19.

Figura 17 - Modelo para otimização da voluta

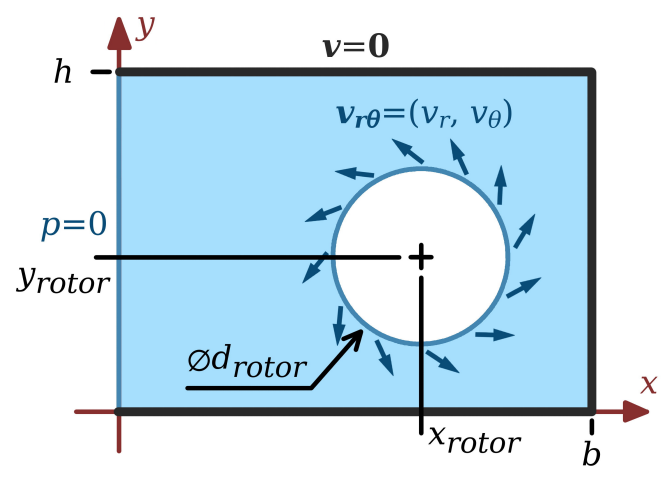

Fonte: Própria

Figura 18 - Voluta otimizada

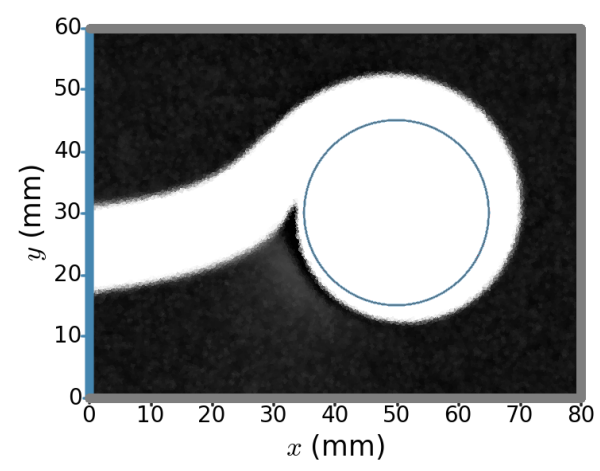

Fonte: Própria

\subsection{Experimentos com a bomba Tesla de CDs}

Os experimentos com a bomba Tesla de CDs foram realizados com rotações de 300 a 800 rpm (figura 19).

Figura 19 - Curva de pressão $\times$ vazão volumétrica obtida experimentalmente e sobreposta aos seus valores de simulações do modelo da figura 10

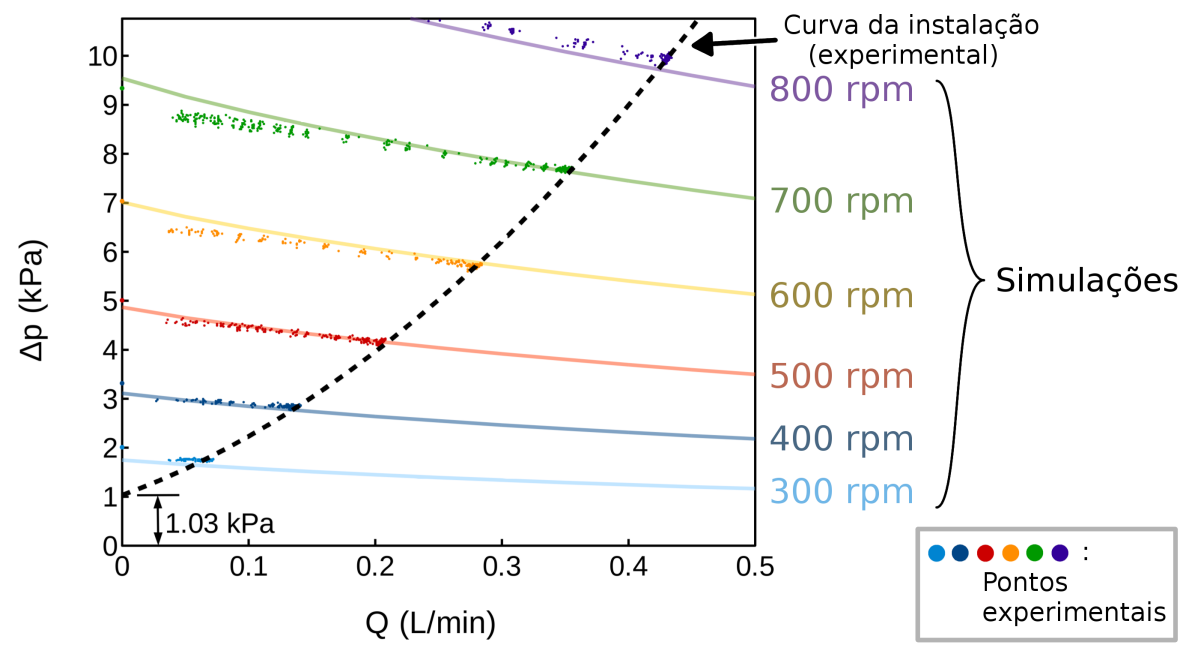

Fonte: Própria 
Para se ter uma visão mais ampla das curvas da bomba Tesla na figura 19 obtidas em simulação, o gráfico da figura 21 se estende até $4 \mathrm{~L} / \mathrm{min}$.

Figura 20 - Curvas de pressão $\times$ vazão volumétrica estendidas, obtidas em simulações do modelo da figura 10

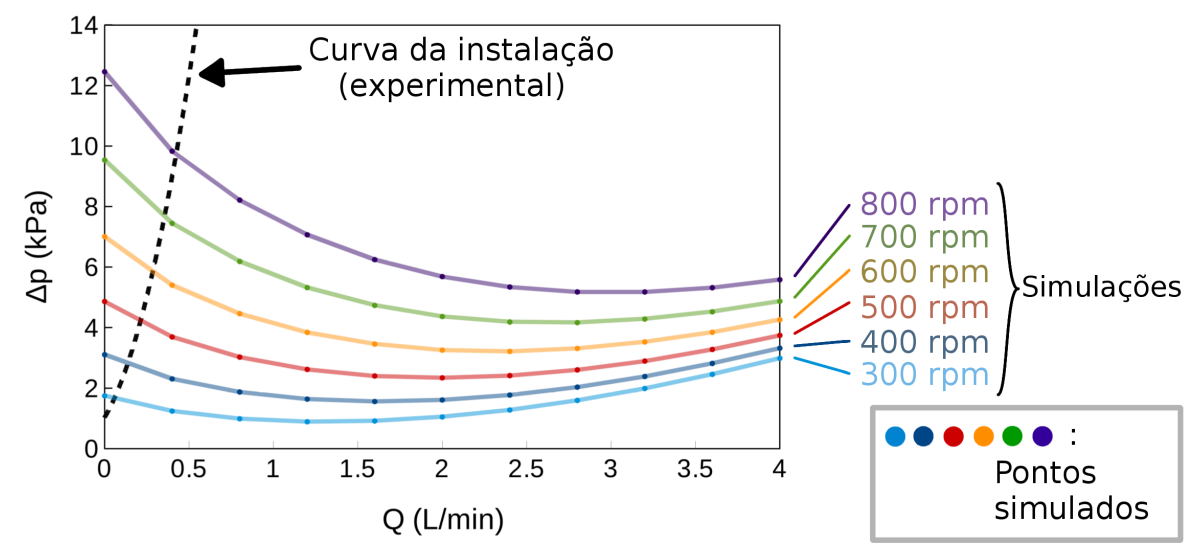

Fonte: Própria

\subsection{Experimentos com a mini bomba Tesla}

Como o sensor de vazão não conseguia medir com a mini bomba Tesla na rotação utilizada na otimização (500 rpm), utilizaram-se as rotações $2254 \mathrm{rpm}$ e $3154 \mathrm{rpm}$ (figura 21).

Figura 21 - Curva de pressão $\times$ vazão volumétrica obtida experimentalmente para a mini bomba Tesla

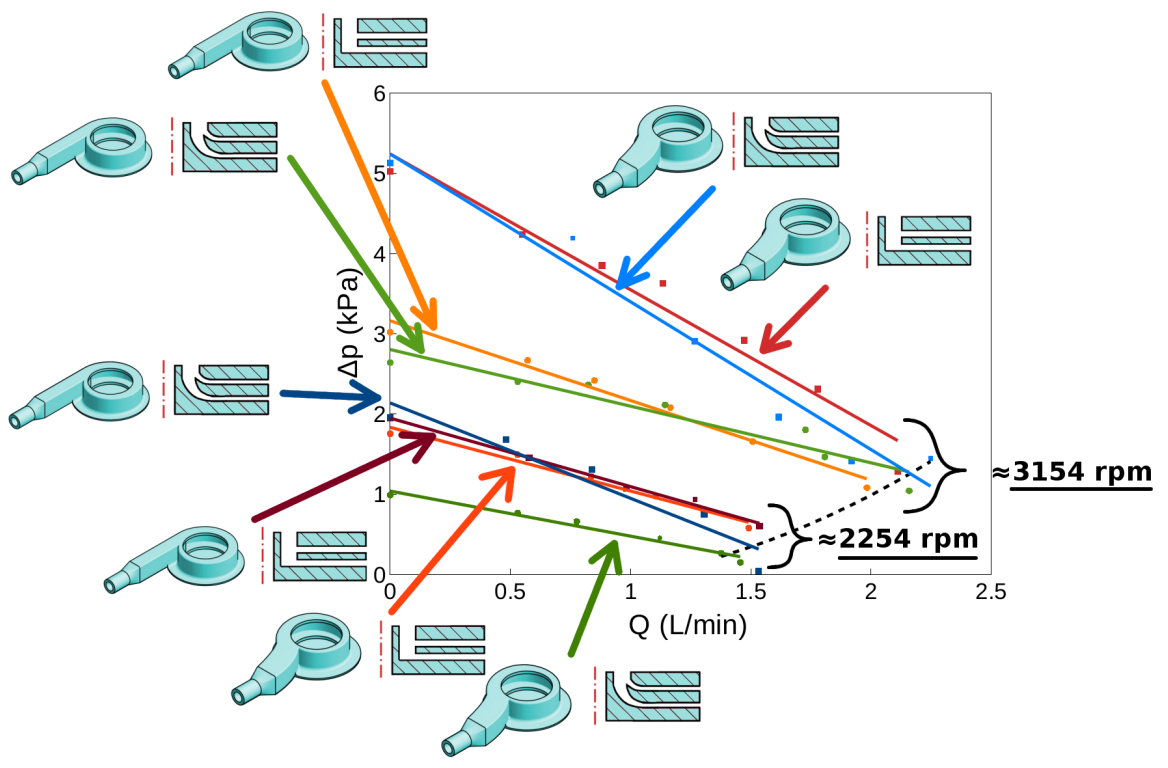

Fonte: Própria

\section{Conclusões}

O projeto da bomba Tesla de CDs foi realizado por meio de análise paramétrica do espaçamento entre discos, utilizando-se um projeto de voluta convencional. As medições experimentais resultaram coerentes com as simulações do modelo simplificado de dois discos da bomba Tesla. Os projetos do rotor e da voluta de uma bomba Tesla foram otimizados considerando-se água em regime permanente e escoamento laminar. Os resultados experimentais mostram que as configurações otimizadas demonstraram menor pressão do que as configurações não-otimizadas, o 
que pode se dever a não imposição de um ponto de operação (carga manométrica) na otimização topológica.

\section{Referências}

ALMEIDA, S. R. M.; PAUlinO, G. H.; SILVA, E. C. N. Layout and material gradation in topology optimization of functionally graded structures: a global-local approach. Structural and Multidisciplinary Optimization, v. 42, n. 6, p. 855-868, Dec 2010.

AMESTOY, P. R.; DUFF, I. S.; KOSTER, J.; L'EXCELLENT, J.-Y. A fully asynchronous multifrontal solver using distributed dynamic scheduling. SIAM Journal on Matrix Analysis and Applications, v. 23, n. 1, p. 15-41, 2001.

BEHBAHANI, M.; BEHR, M.; HORMES, M.; STEINSEIFER, U.; ARORA, D.; CORONADO, O.; PASQUALI, M. A review of computational fluid dynamics analysis of blood pumps. v. 20, p. $363-397,082009$.

BENDSØE, M. P.; KIKUCHI, N. Generating optimal topologies in structural design using a homogenization method. Computer Methods in Applied Mechanics and Engineering, v. 71, n. 2, p. $197-224,1988$.

BORRVALL, T.; PETERSSON, J. Topology optimization of fluids in stokes flow. International Journal for Numerical Methods in Fluids, John Wiley \& Sons, Ltd., v. 41, n. 1, p. 77-107, 2003.

BREITER, M. C.; POHLHAUSEN, K. Laminar flow between two parallel rotating disks. Wright-Patterson AFB-O: [s.n.], 1962. 81 p. Aeronautic Research Laboratory Office.

DODSWORTH, J.; GROULX, D. Operational parametric study of a tesla pump including vibration effects. In: ASME-ATI-UIT Conference on Thermal Energy Systems: Production, Storage, Utilization and the Environment. Napoly: [s.n.], 2015.

DODSWORTH, L. Operational parametric study of a prototype tesla pump. Dissertação (Mestrado) — Dalhousie University, 2016.

DUAN, X.; LI, F.; QIN, X. Topology optimization of incompressible navier-stokes problem by level set based adaptive mesh method. Computers \& Mathematics with Applications, v. 72, n. 4, p. $1131-1141,2016$.

ENGIN, T.; ÖZDEMIR, M.; ÇEŞMECI, Ş. Design, testing and two-dimensional flow modeling of a multiple-disk fan. Experimental Thermal and Fluid Science, v. 33, n. 8, p. 1180 - 1187, 2009.

FARRELL, P. E.; HAM, D. A.; FUNKE, S. W.; ROGNES, M. E. Automated derivation of the adjoint of high-level transient finite element programs. SIAM Journal on Scientific Computing, v. 35, n. 4, p. C369-C393, 2013.

FOO, S.; TAN, W. C.; SHARIL, M. Development of tesla turbine for green energy applications. In: LINKÖPING UNIVERSITY ELECTRONIC PRESS. 2nd NCMER 2010. National Conference in Mechanical Engineering Research and Postgraduate Studies. [S.l.], 2010.

FOSTER, M. The potential of a Tesla type device as a non pulsatile blood pump. Tese (Doutorado) - Middlesex University, 2006. 
FULLER, H. J. Wind turbine for generation of electric power. US Patent 7,695,242. 1913.

HO-YAN, B. P. Tesla turbine for pico hydro applications. Guelph Engineering Journal, v. 4, p. 1-8, 2011.

IZRAELEV, V.; WEISS, W. J.; FRITZ, B.; NEWSWANGER, R. K.; PATERSON, E. G.; SNYDER, A.; MEDVITZ, R. B.; CYSYK, J.; PAE, W. E.; HICKS, D. et al. A passively-suspended tesla pump left ventricular assist device. ASAIO journal (American Society for Artificial Internal Organs: 1992), NIH Public Access, v. 55, n. 6, p. 556, 2009.

LAMPART, P.; KOSOWSKI, K.; PIWOWARSKI, M.; JĘDRZEJEWSKI, Ł. Design analysis of tesla micro-turbine operating on a low-boiling medium. Polish Maritime Research, v. 16, n. Special, p. 28-33, 2009.

LOBANOFF, V. S.; ROSS, R. R. Centrifugal pumps: design and application. 2. ed. [S.l.]: Elsevier, 2013.

LOGG, A.; MARDAL, K.-A.; WELLS, G. Automated solution of differential equations by the finite element method: The FEniCS book. Berlin: Springer, 2012. v. 84.

MedvitZ, R. B.; BOGER, D. A.; IZRAELEV, V.; ROSEnBERG, G.; PATERSON, E. G. Computational fluid dynamics design and analysis of a passively suspended tesla pump left ventricular assist device. Artificial Organs, Blackwell Publishing Inc, v. 35, n. 5, p. 522-533, 2011.

OSHER, S.; SETHIAN, J. A. Fronts propagating with curvature-dependent speed: algorithms based on hamilton-jacobi formulations. Journal of computational physics, Elsevier, v. 79, n. 1, p. 12-49, 1988.

PODERGAJS, M. The tesla turbine. In: Seminar, University of Ljubljana, Faulty of Mathematic and Phsics. [S.l.: s.n.], 2011.

RAJE, A.; SINGH, B.; CHURAI, R.; BORWANKAR, P. A review of tesla turbine. International Journal of Mechanical Engineering and Technology, v. 6, n. 10, p. 28-31, 2015.

REY LADINO, A. F. Numerical simulation of the flow field in a friction-type turbine (Tesla turbine). Dissertação (Diploma thesis) - Institute of Thermal Powerplants, Vienna University of Technology, 2004.

RICE, W. Tesla turbomachinery. 1991.

RODDY, P. J.; DARBY, R.; MORRISON, G. L.; JENKINS, P. E. Performance characteristics of a multiple-disk centrifugal pump. Journal of Fluids Engineering, v. 109, n. 1, p. 51 - 57, 1987.

TESLA, N. Fluid propulsion. US Patent 1,061,142. 1913.

. Turbine. US Patent 1,061,206. 1913.

1921. Improved process of and apparatus for production of high vacua. GB Patent 179,043. Improvements in the construction of steam and gas turbines. GB Patent 186,082. 1921. TUZSON, J. Centrifugal pump design. [S.l.]: John Wiley \& Sons, 2000.

VAFAI, K. Handbook of porous media. 2. ed. [S.l.]: Crc Press, 2005. 
WÄCHTER, A.; BIEGLER, L. T. On the implementation of an interior-point filter line-search algorithm for large-scale nonlinear programming. Mathematical programming, Springer, v. 106, n. 1, p. 25-57, 2006.

YU, H. Flow design optimization of blood pumps considering hemolysis. Tese (Doutorado) Magdeburg, Universität, Diss., 2015, 2015. 
Title: Design, fabrication and characterization of a Tesla pump

Abstract - Tesla devices consist of rotating disks (without blades), whose operation is based on the boundary layer effect (i.e., viscous friction forces acting on the fluid and Coandă effect). According to this working principle, the flow becomes continuous and nonpulsatile. The objective of this project is to design, fabricate and characterize a Tesla pump aiming to improve its efficiency and power. The Tesla pump has various applications, but the efficiency of its operation is quite low, which makes room for the optimization of its design. This project is based on the optimization of the rotor and the volute of a Tesla pump operating with a newtonian fluid in laminar flow. The operation of the Tesla pump is simulated by using the Finite Element Method in the FEniCS platform and validated with the software ANSYS ${ }^{\circledR}$ CFX. The topology optimization is implemented in the FEniCS platform by using the dolfin-adjoint library for sensitivity (derivative) calculation and the IPOPT algorithm for optimization. Two prototypes are fabricated and characterized experimentally, consisting of a Tesla pump composed of CDs (polycarbonate discs used as digital data storage) as the rotor disks; and a prototype designed by the topology optimization method (fabricated by additive manufacturing).

Keywords - Centrifugal pump, Fluid dynamics (Simulation), Finite element method, Topology methods (Optimization).

\section{Diego Hayashi Alonso}

Graduação em Engenharia Mecatrônica na Escola Politécnica da Universidade de São Paulo (2017). Artigo elaborado para o Trabalho de Conclusão de Curso (PMR2550, Projeto de Conclusão de Curso II), sob orientação do Prof. Dr. Emílio Carlos Nelli Silva (Laboratório de Otimização de Sistemas Multifísicos) e simplificado e adaptado para o formato da revista Mecatrone. 